\title{
Resonant purification of mixed states for closed and open quantum systems*
}

\author{
Raffaele Romand \\ Department of Mathematics, Iowa State University, Ames IA 50011, USA
}

\begin{abstract}
Pure states are fundamental for the implementation of quantum technologies, and several methods for the purification of the state of a quantum system $S$ have been developed in the past years. In this letter we present a new approach, based on the interaction of $S$ with an auxiliary system $P$, having a wide range of applicability. Considering two-level systems $S$ and $P$ and assuming a particular interaction between them, we prove that complete purifications can be obtained under suitable conditions on the parameters characterizing $P$. Using analytical and numerical tools, we show that the purification process exhibits a resonant behavior in both the cases of system isolated from the external environment or not.
\end{abstract}

PACS numbers: 02.30.Yy, 03.65.Ud, 03.67.-a

Keywords: Purification of mixed states, quantum control, non-Markovian dynamics

Introduction. - The quantum theories of information and computation suggest that quantum mechanics based devices could highly outperform the corresponding classical apparatuses in several fields [1]. At the heart of this qualitative improvement there are the quantum correlations called entanglement, absent in classical systems. In full generality, pure states are needed for the implementation of quantum processing. These states, represented by projectors on the Hilbert space associated to the system, do not contain classical correlations.

Unfortunately, when a quantum system interacts with its surrounding environment, it loses its relevant properties, since it is subject to a dissipative evolution leading to decoherence and irreversible transitions from pure to mixed states (that is probabilistic superpositions of pure states). To avoid this problem, it is fundamental to decouple as much as possible system and environment or, whenever this is not possible, to study methods for counteracting the environmental action, thus preserving the entanglement and restoring the purity of the states (the so-called purification process).

The study of methods for the purification of a quantum state, that is the transition to less mixed states, provides a rich field of investigation. Several strategies have already been proposed, especially in the context of entanglement purification [2, 3]. They rely on two mechanisms: measurement or cooling procedures (see [4, 5, 6, 67, 8] and references therein). From a general viewpoint, it is necessary to consider how it is possible to affect the dynamics of the system, and what states can be attained during its time evolution. In this context, one assumes that some dynamical parameters can be externally modified. They are called controls and, by means of them, specific transitions can be induced (see [9, 10] and references therein).

Usually, the controls are assumed to affect the dynamics through the Hamiltonian of the system. The corresponding models are called coherent control methods as the controls enter the coherent part of the dynamics, and their properties have been widely investigated. In particular, it has been proven that quantum states cannot be purified by using coherent control, both in closed and open systems dynamics, whether the controls are fixed at the beginning [11, 12]. To overcome this difficulty, several solutions have been proposed, as the use of an indirect measurement, performed on the system. In some schemes, the outcomes of the measurement are used to continuously update the controls (quantum feedback, see e.g. 13, 14]), and state purifications can be realized. Another possibility is represented by the cooling techniques: they rely on a constructive interplay of coherent control and specific dissipative mechanisms, leading to a purity increase in the system at the bath expenses.

Recently, a different control scheme has been developed, in which the controls are fixed a priori, and the assumption of coherent control is relaxed [15]. This noncoherent control protocol relies on the use of an initially uncorrelated auxiliary system, the probe $P$, that is put in interaction with the relevant system $S$ and discarded at the end of the procedure. The controls $u$ enter the dynamics through the initial state of the probe, $\rho_{P}(0)=\rho_{P}(u)$, and the ability to modify the states of the target system depends on the correlations between system and probe, generated by the interaction. The dynamics of the system is given by

$$
\rho_{S}(t, u)=\operatorname{Tr}_{P} \rho_{T}(t)
$$

with

$$
\rho_{T}(t)=\gamma_{t}\left[\rho_{S}(0) \otimes \rho_{P}(u)\right],
$$

where $T$ denotes the composite system $S+P$, and $\gamma_{t}$ its time evolution. If $T$ is a closed system, $\gamma_{t}$ is generated by the Liouville operator

$$
\dot{\rho}_{T}(t)=-i\left[H_{T}, \rho_{T}(t)\right],
$$

where $H_{T}=H_{S}+H_{P}+H_{I}$ is the total Hamiltonian, containing the free contributions $H_{S}$ and $H_{P}$ and the interaction term $H_{I}$. In principle, there is some freedom in the choice of the operators $H_{P}$ and $H_{I}$, because different probe systems could be considered. 
In this letter we prove that the non-coherent control protocol provides an alternative approach to the purification of a qubit system. For a particular choice of the free dynamics of the probe and its interaction with the system, we prove that this control scheme leads to complete purifications of the maximally mixed state also in the pessimistic case of weak interaction between $S$ and $P$, if there is not a surrounding environment. By using both analytical and numerical tools, we find that the purification process has a resonant behavior depending on the energy difference between the two systems. Finally, by considering the worst-case isotropic decoherence, we show how our results are affected by the action of the external environment.

We limit our attention to two-dimensional $S$ and $P$. As a measure of the purity of a state $\rho_{S}$ we consider the von Neumann distance of this state from the maximally mixed state $\mathbb{I} / 2$,

$$
\pi=\sqrt{2 \operatorname{Tr}\left(\rho_{S}-\frac{\mathbb{I}}{2}\right)^{2}}=\sqrt{2 \operatorname{Tr}\left(\rho_{S}^{2}\right)-1}
$$

where a convenient scale factor 2 has been included, such that $0 \leqslant \pi \leqslant 1, \pi=0$ if and only if $\rho_{S}$ is the maximally mixed state, and $\pi=1$ if and only if $\rho_{S}$ is a pure state, $\rho_{S}^{2}=\rho_{S}$.

We introduce a Bloch vector representation for the states,

$$
\rho_{S}(t)=\frac{1}{2}\left(\mathbb{I}+\vec{s}(t) \cdot \vec{\sigma}^{S}\right), \quad \rho_{P}(t)=\frac{1}{2}\left(\mathbb{I}+\vec{p}(t) \cdot \vec{\sigma}^{P}\right)
$$

with $\vec{s}(t), \vec{p}(t)$ real vectors satisfying $\|\vec{s}(t)\|=\|\vec{p}(t)\| \leqslant 1$ and $\vec{\sigma}^{S}, \vec{\sigma}^{P}$ the vectors of Pauli matrices in $S$ and $P$ respectively. The time-dependent purity becomes

$$
\pi(t)=\|\vec{s}(t)\|,
$$

and a purification process amounts to an increase of $\pi(t)$.

For coherent control, $\dot{\rho}_{S}(t)=-i\left[H_{S}(u), \rho_{S}(t)\right]$, therefore

$$
\dot{\pi}(t)=2 \pi^{-1}(t) \operatorname{Tr}\left(\rho_{S}(t) \dot{\rho}_{S}(t)\right)=0,
$$

and the purity of the initial state is not modified, as it has been remarked before.

Purification by means of a probe. - If $T$ is a closed system, the operator $\gamma_{t}$ in (2) is given by

$$
\gamma_{t}[\cdot]=e^{-i H_{T} t} \cdot e^{i H_{T} t} .
$$

In general, it is possible to explore the purification process by using a Cartan decomposition,

$$
e^{-i H_{T} t}=L_{1} e^{a t} L_{2}
$$

where $L_{1}, L_{2}$ are local contributions and $a=c_{x} \sigma_{x}^{S} \otimes$ $\sigma_{x}^{P}+c_{y} \sigma_{y}^{S} \otimes \sigma_{y}^{P}+c_{z} \sigma_{z}^{S} \otimes \sigma_{z}^{P}$, with $c_{x}, c_{y}, c_{z} \in \mathbb{R}$. Since the local transformations do not affect the purification process, it is possible to classify all the possible cases by means of $c_{x}, c_{y}$ and $c_{z}$. In fact, the entangling capability of the operator (9), at the heart of the non-coherent control model, depends on these coefficients. By using the expression of $\vec{s}(t)$ derived in [15], we find that evolutions leading to an increase of the purity of the initial state are possible only if at least two different coefficients $c_{i}$ do not vanish. This condition is weaker than the accessibility and controllability criteria, thus systems that are neither accessible nor controllable can exhibit purification of mixed states. Moreover, it is possible to prove that whenever the maximally mixed state can be completely purified, the same is true for an arbitrary mixed state.

Our aim in this work is to discuss some relevant features of the purification process under the incoherent control protocol, rather than to systematically study all the possible cases. Moreover, we want to analyze this process in terms of the relevant physical quantities entering the dynamics (the characteristic energies of $S$ and $P$ ), rather than in terms of the $c_{i}$ parameters. Therefore we consider a particular model by assuming $H_{S}=\omega_{s} \sigma_{z}^{S}$, $H_{P}=\omega_{p} \sigma_{z}^{P}$ and $H_{I}=g \sigma_{x}^{S} \otimes \sigma_{x}^{P}$, where $\omega_{s}$ and $\omega_{p}$ are the characteristic energies in $S$ and $P$, and $g$ is the coupling constant. The propagator for the total system can be directly computed as

$$
\begin{aligned}
e^{-i H_{T} t} & =\frac{1}{2} \cos (\alpha t)\left(\mathbb{I} \otimes \mathbb{I}+\sigma_{z}^{S} \otimes \sigma_{z}^{P}\right)+ \\
& +\frac{1}{2} \cos (\beta t)\left(\mathbb{I} \otimes \mathbb{I}-\sigma_{z}^{S} \otimes \sigma_{z}^{P}\right)+ \\
& -i \frac{a}{2 \alpha} \sin (\alpha t)\left(\sigma_{z}^{S} \otimes \mathbb{I}+\mathbb{I} \otimes \sigma_{z}^{P}\right)+ \\
& -i \frac{b}{2 \beta} \sin (\beta t)\left(\sigma_{z}^{S} \otimes \mathbb{I}-\mathbb{I} \otimes \sigma_{z}^{P}\right)+ \\
& -i \frac{g}{2 \alpha} \sin (\alpha t)\left(\sigma_{x}^{S} \otimes \sigma_{x}^{P}-\sigma_{y}^{S} \otimes \sigma_{y}^{P}\right)+ \\
& -i \frac{g}{2 \beta} \sin (\beta t)\left(\sigma_{x}^{S} \otimes \sigma_{x}^{P}+\sigma_{y}^{S} \otimes \sigma_{y}^{P}\right),
\end{aligned}
$$

where

$$
\begin{aligned}
\bar{\omega} & =\omega_{s}+\omega_{p}, & \alpha & =\sqrt{\bar{\omega}^{2}+g^{2}}, \\
\delta \omega & =\omega_{s}-\omega_{p}, & \beta & =\sqrt{(\delta \omega)^{2}+g^{2}} .
\end{aligned}
$$

Following the previous discussion, it is not restrictive to choose the maximally mixed state $\rho_{S}(0)=\mathbb{I} / 2$ as initial state for the system $S$. The initial state of the composite system is given by $\rho_{T}(0)=\rho_{S}(0) \otimes \rho_{P}(u)$. Denoting by $\vec{s}(0)=(0,0,0)$ and $\vec{p}(u)=\left(p_{x}, p_{y}, p_{z}\right)$ the Bloch vector representations of the initial states $\rho_{S}(0)$ and $\rho_{P}(u)$, we can compute $s_{i}=\operatorname{Tr}\left(\rho_{T}(t) \sigma_{i}^{S} \otimes \mathbb{I}\right)$ for $i=x, y, z$, with $\rho_{T}(t)$ from (8),

$$
\begin{aligned}
& s_{x}(t)=s_{y}(t)=0 \\
& s_{z}(t)=p_{z} g^{2}\left(\frac{1}{\beta^{2}} \sin ^{2}(\beta t)-\frac{1}{\alpha^{2}} \sin ^{2}(\alpha t)\right) .
\end{aligned}
$$




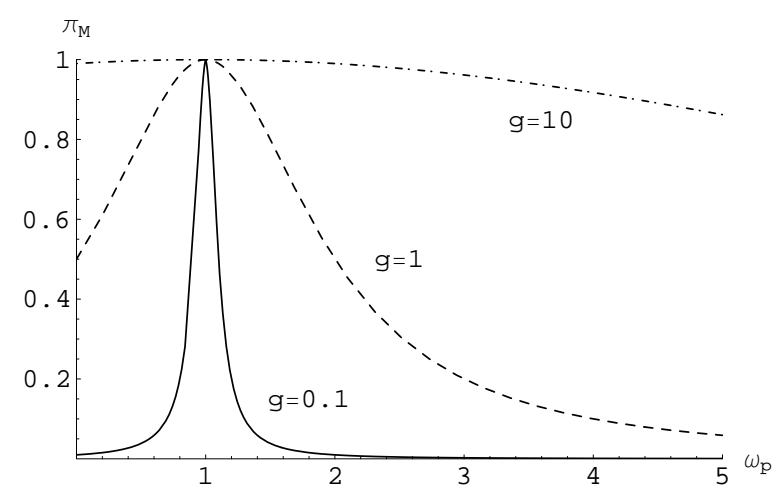

FIG. 1: Maximal attainable purification $\pi_{M}$ for the maximally mixed state in several regimes. The plots exhibit a resonance about $\omega_{p}=\omega_{s}$ in all the cases: dominant interaction (dot-dashed line), interaction and free contribution of the same strength (dashed line), weaker interaction (solid line). The value $\omega_{s}=1$ has been considered.

As the $x$ and $y$ components are constant, the system is not accessible nor controllable. The purity of the state is given by $\pi(t)=\left|s_{z}(t)\right|$ and it is a combination of oscillating functions. After some manipulations, it can be written in the form

$$
\begin{aligned}
s_{z}(t) & =p_{z} g^{2}\left[\frac{\alpha^{2}-\beta^{2}}{2 \alpha^{2} \beta^{2}}(1-\cos (\alpha+\beta) t \cos (\alpha-\beta) t)+\right. \\
& \left.-\frac{\alpha^{2}+\beta^{2}}{2 \alpha^{2} \beta^{2}} \sin (\alpha+\beta) t \sin (\alpha-\beta) t\right] .
\end{aligned}
$$

We are interested in the maximal amplitude of this function, that we denote by $\pi_{M}$. Unfortunately, it is not possible to give an analytical expression of $\pi_{M}$, unless the interaction between $S$ and $P$ is weak, that is $g \ll \omega_{s}, \omega_{p}$. In this case

$$
\pi_{M} \approx \frac{\left|p_{z}\right| g^{2}}{\left(\omega_{s}-\omega_{p}\right)^{2}+g^{2}}
$$

by using the definitions in (11), and the maximal purification amplitude exhibits a Cauchy-Lorentz resonance for $\omega_{p}=\omega_{s}$. The transition from the maximally mixed state to a pure state is obtained by choosing $\left|p_{z}\right|=1$. In Fig. 1 we present the results of a numerical analysis of $\pi_{M}$, showing the behavior of the resonance curve for increasing values of the coupling constant $g$. As intuition could suggest, the ability of inducing specific transitions in the system $S$ by means of $P$ increases with the strength of the coupling between $S$ and $P$.

Purification in open system dynamics. - The incoherent control protocol can be effectively used to increase the purity of the states of an open system, counteracting the decohering effects of the environment. To show this, we adopt the previous model for the free dynamics of the composite system $S+P$, and we add a dissipative contribution:

$$
\dot{\rho}_{T}(t)=-i\left[H_{T}, \rho_{T}(t)\right]+\mathcal{D}\left[\rho_{T}(t)\right],
$$

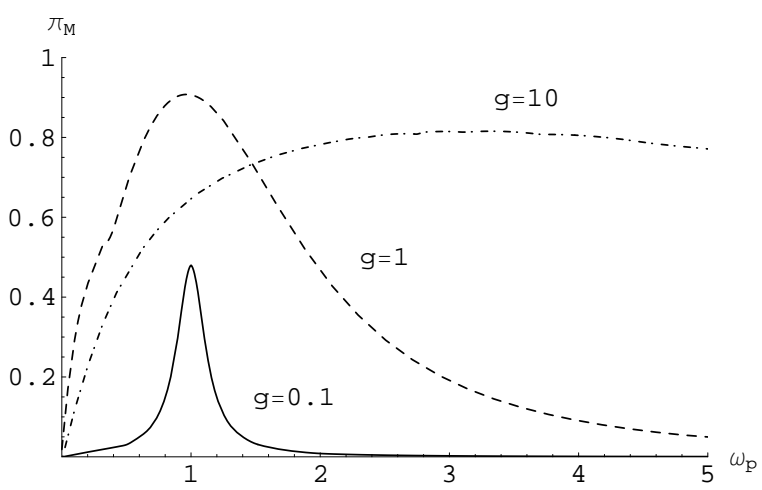

FIG. 2: Maximal attainable purification $\pi_{M}$ for the maximally mixed state, with isotropic decoherence. In general $\pi_{M}$ is decreased by the environmental action, and it still exhibits a resonant behavior. We observe that the peak is about $\omega_{s}=\omega_{p}$ only when the interaction is weaker or comparable to the free contribution, otherwise it moves to $\omega_{p}>\omega_{s}$. We have assumed $\omega_{s}=1$ and $\gamma_{s}=\gamma_{p}=0.01$.

where we assume that the additional term is a Lindblad contribution representing isotropic decoherence,

$$
\begin{aligned}
\mathcal{D}\left[\rho_{T}(t)\right] & =\gamma_{s}\left(\sum_{i} \sigma_{i}^{S} \otimes \mathbb{I} \rho_{T}(t) \sigma_{i}^{S} \otimes \mathbb{I}-\rho_{T}(t)\right)+ \\
& +\gamma_{p}\left(\sum_{i} \mathbb{I} \otimes \sigma_{i}^{P} \rho_{T}(t) \mathbb{I} \otimes \sigma_{i}^{P}-\rho_{T}(t)\right),
\end{aligned}
$$

where $\gamma_{s}, \gamma_{p}$ are the decay rates for $S$ and $P$ respectively, and we neglect any environmental coupling between system and probe.

The results of our numerical analysis are shown in Fig. 2 Also in this case the best choice of the probe is $\left|p_{z}\right|=1$. The general effect of the interaction with the surrounding environment is a decrease of $\pi_{M}$ depending on $g$. By comparing Fig. 1 and Fig. 2] we notice that this effect is suppressed when $g$ is of the order of $\omega_{s}$ and $\omega_{p}$, stronger otherwise. We observe that the peak of the resonance moves from $\omega_{p}=\omega_{s}$ when the coupling constant $g$ is dominant. Moreover, the dissipative dynamics produces a drastic decrease of $\pi_{M}$ for $\omega_{p} \rightarrow 0$.

These effects can be understood by analyzing the different contributions to the dynamics of $S$. We can identify three time scales. Two of them characterize the nonMarkovian dynamics of $S$, depending on the interaction with $P$. From (13), they are given by $(\alpha+\beta)^{-1}$ (fast oscillations) and $(\alpha-\beta)^{-1}$ (slow oscillations). Finally, there is the characteristic time for the damping induced by the environment, given by $\gamma_{s}^{-1}$.

In order to have an effective purification, the environment induced relaxation should be negligible on a time scale of the order of the slow oscillations, that is $\gamma_{S}^{-1} \gg(\alpha-\beta)^{-1}$, otherwise the purification process is weakened by dissipation. Differently from the closed system case, the purification process is not improved by increasing the strength of the coupling between $S$ and $P$, 


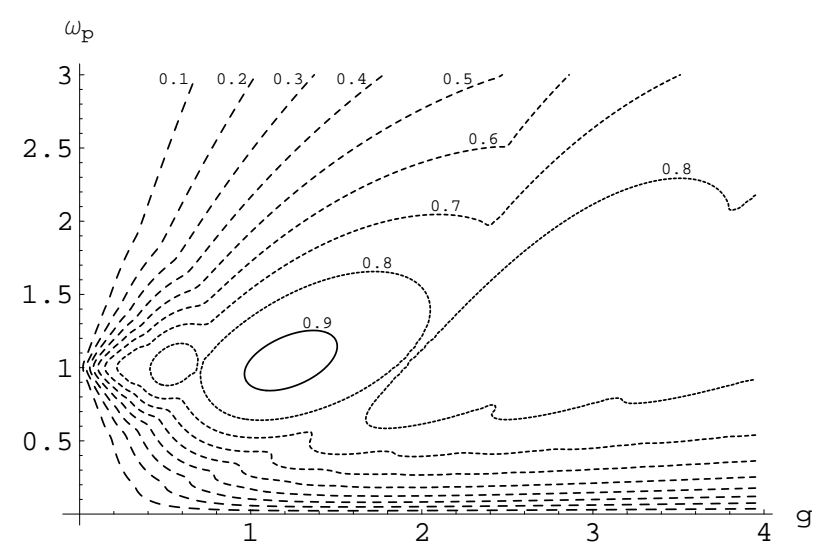

FIG. 3: Contour plot of the maximal attainable purification $\pi_{M}$ in the $g-\omega_{P}$ plane, for the maximally mixed state, with isotropic decoherence. The dynamical parameters are fixed as in Fig. 2 The global maximum is given by $\pi_{M} \approx 0.92$, with $g \approx 1.2$ and $\omega_{p} \approx 1.0$.

since in this case $\alpha \approx \beta \approx g$ and then $(\alpha-\beta)^{-1} \rightarrow+\infty$. In particular, this explains the decrease of $\pi_{M}$ when $g$ is increased, in the open system case. In other words, by increasing $g$ the purification time becomes large and the dissipative effects come into play. Conversely, in the closed system case the purification process takes advantage of a large $g$ since a large purification time does not represent a problem.

A similar result holds for $\omega_{p} \rightarrow 0$ since $\alpha \approx \beta$ as well. This explains the fast decrease of $\pi_{M}$ near $\omega_{p}=0$. Note that the same pattern is expected for $\omega_{p} \rightarrow+\infty$, assuming that $g \gg \omega_{s}$, even if this is not apparent from Fig. 2

In Fig. 3 we present the contour plot of $\pi_{M}$ in the $g-\omega_{P}$ plane for a particular choice of the dynamical parameters. The surface levels suggest a non trivial relation among $\pi_{M}, g$ and $\omega_{p}$, in particular there are several local maxima.

Conclusions. - We have explored the purification process of a quantum system $S$ based on its interaction with an auxiliary system $P$, in both the closed and open system frameworks. We have considered the case of twolevel systems $S$ and $P$, and assumed particular forms of the free dynamics, of the interaction between $S$ and $P$, and of their interaction with the environment. We have found that the purification process exhibits a resonant behavior that depends on the energy difference between the system and the probe. When the interaction between $S$ and $P$ is weak, this resonance is described by a Lorentz-Cauchy curve. We have shown that complete purifications are possible in the closed system case. In the open system case, the attainable purity is reduced, and it reaches a maximum for optimal values of the parameters characterizing the dynamics.

The probe-mediated protocol differs from the existing purification techniques, based on measurement or cooling, and it has a different range of applicability. From this point of view, it complements the existing methods. Usually, in measurement-based schemes, an indirect measurement is needed to implement feedback control. In our case, measurement does not enter the procedure (unless the initial state of $P$ is prepared by a measurement), and there is not feedback. Similarly to the cooling procedures, in our approach the aim is to transfer the purity between different systems. However, this transition has a completely different origin, since it depends on the non-Markovian dynamics of $S$, not on a particular dissipative mechanism. Therefore, the method is effective with any kind of dissipation at work, and then it is of wider applicability. Moreover, in our approach there is not coherent control on $S$.

The results obtained with the particular model described in this work are not exceptional. In fact, we have found numerical evidence of the resonance in the $\mathrm{pu}-$ rification process with more general Hamiltonian terms. Using $H_{S}=\vec{n} \cdot \vec{\sigma}^{S}, H_{P}=\vec{m} \cdot \vec{\sigma}^{P}$, and varying the vectors $\vec{n}$ and $\vec{m}$, we have observed that the purification of the maximally mixed state is optimal when $\vec{p}(u)=\vec{m}$. Further analysis will explore these more general cases as well as general environments. Complete purifications are expected also in the open system case, when dissipative models different from isotropic decoherence are considered.

* This material is based upon work supported by the National Science Foundation under Grant No. 0237925

† Electronic address: rromano@iastate.edu

[1] M.A. Nielsen and I.L. Chuang, Quantum Computation and Quantum Information, Cambridge, 2000

[2] C.H. Bennett et al., Phys. Rev. Lett. 76, 722 (1996)

[3] J.I. Cirac, A.K. Ekert and C. Macchiavello, Phys. Rev. Lett. 82, 4344 (1999)

[4] H. Nakazato, T. Takazawa and K. Yuasa, Phys. Rev. Lett. 90, 060401 (2003)

[5] J. Combes and K. Jacobs, Phys. Rev. Lett. 96, 010504 (2006)

[6] H.M. Wiseman and J.F. Ralph, New J. Phys. 8, 90 (2006)

[7] D.J. Tannor and A. Bartana, J. Phys. Chem. A 103, 10359 (1999)

[8] S.E. Sklarz, D.J. Tannor and N. Khaneja, Phys. Rev. A 69, 053408 (2004)

[9] V. Ramakrishna, M. V. Salapaka, M. Dahleh, H. Rabitz and A. Peirce, Phys. Rev. A 51, 960 (1995)

[10] F. Albertini and D. D'Alessandro, IEEE Transactions on Automatic Control 48, 1399 (2003)

[11] W. Ketterle and D.E. Pritchard, Phys. Rev. A 46, 4051 (1992)

[12] C. Altafini, J. Math. Phys. 44, 2357 (2003)

[13] H.M. Wiseman and G. J. Milburn, Phys. Rev. Lett. 70, 548 (1993)

[14] S. Mancini, D. Vitali, and P. Tombesi , Phys. Rev. Lett. 80, 688 (1998)

[15] R. Romano and D. D'Alessandro, Phys. Rev. A 73, 022323 (2006) 